\title{
Mathematical Formalisms in Scientific Practice: From Denotation to Model-Based Representation
}

\author{
Axel Gelfert* \\ Department of Philosophy \\ National University of Singapore \\ 3 Arts Link, 117570 Singapore \\ email: axel@gelfert.net
}

June 10, 2010

\begin{abstract}
The present paper argues that 'mature mathematical formalisms' play a central role in achieving representation via scientific models. A close discussion of two contemporary accounts of how mathematical models apply - the DDI account (according to which representation depends on the successful interplay of denotation, demonstration and interpretation) and the 'matching model' account - reveals shortcomings of each, which, it is argued, suggests that scientific representation may be ineliminably heterogeneous in character. In order to achieve a degree of unification that is compatible with successful representation, scientists often rely on the existence of a 'mature mathematical formalism', where the latter refers to a - mathematically formulated and physically interpreted - notational system of locally applicable rules that derive from (but need not be reducible to) fundamental theory. As mathematical formalisms undergo a process of elaboration, enrichment, and entrenchment, they come to embody theoretical, ontological, and methodological commitments and assumptions. Since these are enshrined in the formalism itself, they are no longer readily obvious to either the novice or the proficient user. At the same time as formalisms constrain what may be represented, they also function as inferential and interpretative resources.

Keywords: Representation, denotation, mathematical formalism, inferential utility, scientific models.
\end{abstract}

*Financial support via an AcRF Tier 1 grant (WBS R-106-000-016-112) from the Ministry of Education (MOE) Singapore is gratefully acknowledged. I would like to thank Tarja Knuuttila and Mauricio Suárez for helpful comments on an earlier draft of this paper. 


\section{Introduction}

Idealization, abstraction, and approximation have long been at the heart of much of the physical sciences, and the deliberate construction of scientific models has an only slightly shorter history. And yet there is a sense that the systematic and pervasive deployment of scientific modelling techniques is a fairly recent phenomenon; in some disciplines - especially those that deal with complex systems - scientific models have become the default mode of how to approach scientific problems. As Adam Morton puts it, 'what is new and distinctive in the science of our time is the existence of complex mediating models which themselves have explanatory power and which embody techniques of modelling which can be refined and passed down to successor models, even though the models never themselves can function as background theories' [Morton 1993, p. 664]. In the present paper, I focus on the role of mathematics in this process; in particular, I shall argue that 'mature mathematical formalisms' embody inferential strategies and theoretical commitments that contribute to, and sometimes ensure, the representational success of scientific models.

In the philosophical literature, the success of models continues, for better or worse, to be discussed in representational terms: in short, scientific models represent, and successful models represent successfully. In the present paper, I do not attempt to explain what scientific representation is in general. Instead, I focus on how mathematical models represent reality. In particular, I want to argue for a positive role of mathematical formalisms in achieving representation. The notion of 'mathematical formalism' calls for some clarification. As I understand the term, a mature mathematical formalism is a system of rules and conventions that deploys (and often adds to) the symbolic language of mathematics; it typically encompasses locally applicable rules for the manipulation of its notation, where these rules are derived from, or otherwise systematically connected to, certain theoretical or methodological commitments. As a result, a mature mathematical formalism is imbued with, but not usually reducible to, what Nancy Cartwright has called 'fundamental theory' (where the latter aims at a complete account solely in terms of fundamental laws; see [Cartwright 1983, p. 1]). A given mathematical formalism need neither be unique nor complete (in whatever sense of uniqueness or completeness), though its amenability to a wide range of representational tasks will, of course, count in its favour. In order to avoid misunderstanding, it is perhaps worth pointing out that, as I understand the term, 'mathematical formalism' does not refer to (nor entail any preference with respect to) any particular debate or position within the philosophy of mathematics; my concern in this paper is with how scientists deploy (physically interpreted) mathematical formalisms as inferential and interpretative strategies.

The present paper can be thought of as divided into two parts, Sections 1-3 and Section 4. The first three sections are devoted to a close analysis of the challenges and problems faced by contemporary accounts of scientific representation, especially in relation to mathematical models. Section 4, which makes up roughly the second half of the paper, develops in detail my view that 
mature mathematical formalisms in science function as inferential practices and interpretative resources and play an essential role in achieving representation.

\section{Recent accounts of how models apply}

In the present section, I discuss two recent accounts of how mathematical models represent, each of which is indicative of a certain conception of how mathematical models apply to the world. On the first account, a mathematical model - as stated, say, in the form of a set of equations - represents a target system indirectly, via an intermediate 'matching model' that is posited as isomorphic to the target system. The representational power of a given mathematical model thus resides in its overall ability to 'map' more or less idealised physical situations. The second approach takes as primary the observation that mathematical modelling is a theoretical process that involves different component steps; representation, on this account, is to be understood as the joint outcome of different contributing factors that are by and large on a par with one another.

\subsection{Derivation and matching models}

One strand of the philosophical debate about mathematical models has developed from the perceived indispensability of mathematical entities in scientific explanation. Many explanations, especially in physics, appear to be based essentially on mathematical features, rather than on an appeal to causal factors, which are often abstracted away or otherwise discounted. Philosophers of mathematics have sometimes appealed to nature's apparent amenability to mathematical representation, and to the explanatory power of mathematics, as evidence for Platonism about mathematical entities. For philosophers of science, too, our far-reaching reliance on mathematics as a means for representation and scientific explanations, raises important questions: How do mathematical models represent the world, and how can we hope to gain understanding of the physical world by deploying such models? In the present section, I shall take as my starting point one recent philosophical proposal, due to Christopher Pincock, regarding the question of how mathematical models apply to the world. I shall follow Pincock by beginning with a concrete example, the partial differential equation known as the one-dimensional heat equation, which is used to represent the heat flow along one direction of a material with thermal conductivity $\kappa$, density $\rho$, and specific heat $s$. The temperature distribution $u(x, t)$ at point $x$ and time $t$ may then be described by the partial differential equation

$$
\left(\frac{\kappa}{\rho s}\right)^{2} \frac{\partial^{2}}{\partial x^{2}} u(x, t)=\frac{\partial}{\partial t} u(x, t) .
$$

Whenever we apply a particular equation to a physical phenomenon, we can inquire into (1) the equation's relation to the theory that is presumed to cover the phenomenon in question, and (2) the adequacy of the equation as a representation of the phenomenon. In the present example, this leads to the following 
questions: (1) 'under what conditions are scientists warranted in adding this equation to their 'scientific theory of heat', and (2) 'under what conditions are scientists warranted in using this equation to describe a particular physical system?' [Pincock 2005, p. 68] The former question, in Pincock's terminology, constitutes a search for derivation conditions, while the latter question calls for the specification of application conditions. Let us turn to the problem of derivation conditions first, with the caveat that the specific conditions and assumptions that feature in the context of a derivation will, of course, vary from one example to another. Given that the mathematical details of the derivation of the 1-D heat equation are discussed in Pincock's case study, I shall confine myself to pointing out some of the derivation's general features, especially those that illustrate the kinds of questions that arise from the demand for derivation conditions.

In deriving the equations that are constitutive of a mathematical model, one may, for example, start from empirically established regularities that hold across a wide range of experimental data. ${ }^{1}$ Such regularities are sometimes invoked as phenomenological laws, as, for example, in Hooke's law of applied mechanics. In the case of the heat equation, the relevant phenomenological law is Newton's law of cooling, according to which the rate of heat loss of one body to another is proportional to the difference in temperature between them. The regularities that phenomenological laws describe typically do not hold universally: Hooke's law, which describes the linear elongation of a body (e.g., a metal spring) under an external force (e.g., a weight attached to the spring), is violated once the external force becomes too strong; Newton's law of cooling, which entails that a macroscopic object is cooled most effectively by placing it in a heat bath with minimal (ideally: zero) temperature, neither holds for microscopic systems governed by quantum mechanics, nor for non-classical cooling methods such as thermal annealing. (See ref. [Tarnow 1994].) Such limitations and exceptions are one reason why phenomenological laws are not themselves considered part of fundamental theory, even if, in some cases, phenomenological laws may be derived - often retrospectively - as limiting cases of more fundamental laws. In speaking of phenomenological 'laws', as well as in formulating them in terms of such quantities as the (momentary) 'rate of heat loss', one is already, strictly speaking, extrapolating beyond the empirical findings: any experiment, however elaborately carried out, can only ever measure the finite amount of heat that is transferred over a non-zero period of time, not the momentary rate of heat loss at a given instant.

This distinction between the 'coarse-grained' character of the experimental data (consisting of measurements of finite differences $\Delta x_{1}, \Delta x_{2}, \ldots$ ) and the 'infinitesimal' character of Newton's law, is more than mere hairsplitting. Rather, it points to an important aspect of mathematical modelling, especially where this involves deriving differential equations such as the 1-D heat equation. In virtually all such derivations, a transition from difference equations

\footnotetext{
${ }^{1}$ Other background assumptions will, of course, need to be added as well; this includes certain prescriptions and conventions regarding the interpretation of the equations.
} 
to differential equations requires a limiting procedure, such as taking the limit $\Delta x \rightarrow 0$, thereby replacing measurable differences with unobservable infinitesimal changes. Their ubiquity across so many areas of science should not belie the fact that such limiting procedures raise some very fundamental problems. Apart from the mathematical question of whether the limiting procedure successfully converges or not, there is the fundamental worry that such limiting procedures may cast doubt on whatever representational significance the quantities in question might have had in the first place. Consider the example of the 1-D heat equation. Deriving the partial differential equation, in its form given in equation (1) above, requires, amongst other steps, evaluating the heat loss across an infinitesimal distance $\Delta x$ in the limit $\Delta x \rightarrow 0$. However, given the (undisputed) atomic constitution of matter, and a further fundamental tenet of the theory of heat - namely that heat is simply the average kinetic energy of the constituent atoms - taking the limit $\Delta x \rightarrow 0$ would quickly become meaningless: there is no such thing as the heat at a given point in space, only the heat in a region of space that is large enough to actually accommodate an ensemble of atoms. Yet, for the mathematical derivation of the heat equation it is crucial that it be possible to take the limit - stopping at some finite minimum length $\Delta x>0$ will not do. The problem, then, appears to be this: in deriving the heat equation - an equation which purports to describe the physical phenomenon of heat conduction - we start from an empirically measurable relationship (the proportionality between any finite amount of heat transferred and the temperature difference between two objects), yet we are forced to introduce quantities and employ limiting procedures which no experiment would lead us to think are well-defined. As Pincock puts it, we have here 'an apparent gap between our evidence for the premises of the derivation and the assumptions needed to warrant the steps of the derivation.' [Pincock 2005, p. 70]

How, then, can one justify placing trust in such derivations as are illustrated by the heat equation? One option would be to assess the final result of the derivation - in this case, the heat equation - simply on its own terms, by its empirical performance or its predictive success. However, such an instrumentalist approach would make the success of our derivations, if not a miracle, then certainly a mystery. Derivations play an important part as aides to our understanding of mathematical models and to the resolution of interpretative problems; dismissing the value of actual derivations in favour of brute instrumental success would at the very least be premature. A less radical approach might acknowledge the relevance of the derivation, but regard the latter as a primarily mathematical exercise, which need not lend itself to a physical interpretation at every step. This, in effect, is the position advocated by Pincock, who holds that in deriving mathematical models, we typically move back and forth between a physical attitude - 'which insists that throughout we are talking about physical systems and physical magnitudes' [Pincock 2005, p. 70] - and a mathematical attitude, which views such steps as taking the 'unphysical' limit $\Delta x \rightarrow 0$ as 'involving only mathematical objects' (in this case, infinitesimals). Each attitude alone typically does not suffice: from the perspective of the physical attitude, many steps of a derivation will strictly speaking be false - simply 
because the idealisations and approximations employed are known to be just that: idealisations and approximations, rather than true representations of the full state of affairs - whereas adopting a thoroughgoing mathematical attitude would sever the link that connects the derived model equation to the physical system, or phenomenon, which it is supposed to describe.

The claim that derivations of model equations involve two, prima facie incompatible attitudes, is not intended as a merely descriptive claim about scientific practice. The point is not that some scientists lean more towards one attitude rather than the other, or that there is perpetual vacillation between the two; instead, both are essential components of any complete account of mathematical modelling, and each contributes in distinct ways to the representational capacity of mathematical models. Whether or not a model represents a physical system, or phenomenon, cannot be a question of whether or not it accurately mirrors every single detail; such a criterion would be far too strong, not only because it could hardly ever be met (except in the most simplistic and uninteresting cases), but also because many phenomena - for example in thermodynamics - are quite robust with respect to minor variations (e.g., among microstates). Some degree of idealisation and approximation is thus entirely legitimate, yet, for the reasons described earlier, these are also likely to give rise to steps in the derivation that are incompatible with the physical attitude. At the same time, some approximations or idealisations, while mathematically admissible, may simply be too crude, thereby undermining the representational capacity of the resulting equations. What is needed, then, is a framework which not only accommodates both attitudes in such a way as to assign each its proper place, but which also recognises that both are indispensable - the physical attitude for giving empirical content to what would otherwise be a bare mathematical structure, and the mathematical attitude for ensuring the validity of the intermediate derivational steps.

Pincock attempts to develop such a framework by making mathematical modelling - that is, the successful derivation of model equations that represent a physical system - a two-step process. Given that model equations are typically the outcome of idealisation and abstraction, any attempt to imbue them with representational content retrospectively, in a way that does not respect how they were derived, would seem arbitrary, at best loosely aimed at filling in details that may have been lost in the course of the derivation. Instead of trying to vindicate representation on the basis of a set of (post-derivation) equations, one might look for a more direct criterion for when a model has the potential to represent. One criterion that might make representation seem inevitable, would be the existence of an isomorphism between the model and the physical system or phenomenon it is supposed to represent. ${ }^{2}$ Under this condition, so one might argue, a model would not only represent, it would also represent truly. (On this

\footnotetext{
${ }^{2}$ On closer inspection, however, isomorphism - at least in its naive form - suffers from numerous problems, not least its inability to make sense of the asymmetry of the representation relation: after all, a successful model represents its target system, not vice versa. (On this point, see [Suárez 2003, p. 232f.].) For a discussion of more elaborate proposals in the same vein as isomorphism, see the second half of Section 4.3.
} 
point, see [Pincock 2005b].) However, a model that has been derived via a series of idealisations and approximations will typically not be isomorphic to any real system. In order to nonetheless save the idea that 'representation must involve an isomorphism between some mathematical model and the physical situation represented', Pincock suggests 'that the model described in the derivation (the derivation model) be distinct from the model that is isomorphic to the physical situation (the matching model)'. [Pincock 2005, p. 71] On this account, when dealing with the derivation model $M_{D}$, we are entitled to adopt the mathematical attitude and treat it as a wholly mathematical object, since it is not itself isomorphic to the physical situation $P$, but is instead only indirectly connected to the latter, via its relation to the matching model $M_{M}$.

At first sight this seems to be a mere shift in the burden of proof, since it is now the matching model $M_{M}$, which must stand in the right relation of isomorphy to $P$. There also is the further question of what conditions must be in place for $M_{D}$ to be related to $M_{M}$ in such a way that the former 'inherits', as it were, the representational capacity of the matching model, and what the nature of this relation would have to be. Pincock's suggestion that what is needed is an 'acceptable mathematical transformation' from $M_{D}$ to $M_{M}$, combined with the condition that $M_{M}$ be isomorphic to 'the entire physical situation in all its details and complexities' [Pincock 2005, p. 71], may be unsatisfactory for those interested in general criteria of when a concrete representational device (say, the set of equations that states $M_{D}$ ) does, in fact, function as a faithful (or true) representation. ${ }^{3}$ Whether such universally applicable criteria can ever be found is dubious, though. When, exactly, is a mathematical transformation 'acceptable', and when can a model be said to match a physical situation to the right degree of complexity? These are questions which may have no general answer, requiring instead attention to the specifics of each case. ${ }^{4}$ Here, it suffices to note that, at the very least, the distinction between two kinds of models, $M_{M}$ and $M_{D}$, provides a possible way of reconciling the two competing demands posed by the physical and the mathematical attitude, respectively: to provide a representation of reality, and to do so in a mathematically accessible way.

\subsection{Denotation, demonstration, and interpretation}

An alternative way of approaching the question of how models represent would begin not from the question of how mathematics applies to the world, but from an attempt to analyse the process of mathematical modelling. One such account, to be discussed here, has been proposed by R.I.G. Hughes (1997). According to

\footnotetext{
${ }^{3}$ On the distinction between (mere) epistemic representation and faithful epistemic representation, see [Contessa 2007, p. 54f.]; for a similar distinction between accurate, true, and complete representations, see [Suárez 2004, p. 767f.].

${ }^{4}$ Paul Teller makes a similar point when he argues that any search for intrinsic features of an object that make it a model' is misguided: 'Once this is fully appreciated it becomes clear that we can get on with the project on the strength of a good supply of clear cases of things which are used to represent. These will adequately support study the variety of such uses, the way they function in the scientific enterprise, their interrelations, and so on.' [Teller 2001, p. 397]
} 
this account, the representational capacity of theoretical models is due to the interplay between three components: denotation, demonstration, and interpretation. Denotation (more about which later) accounts for the fact that theoretical elements of a model purport to refer to elements of the physical world; the possibility of demonstration within a theoretical model - taken for granted in any application of mathematical derivation techniques - attests to the fact that models possess an internal dynamic (which allows us to demonstrate theoretical conclusions); interpretation, finally, is what relates demonstrated results back to the physical world. Regarding the earlier discussion of the two challenges any successful account of mathematical models must meet, the triad of denotation, demonstration, and interpretation (henceforth: DDI) suggests the following picture: denotation, as the basic relation whereby certain elements of a model can 'stand for', or 'be a symbol of', elements in the physical world, may explain how physical magnitudes can be 'picked out' by mathematical objects, thereby shedding light on the puzzling fact of the physical world's apparent amenability to mathematical description. Demonstration and interpretation, by contrast, jointly account for the derivation of specific results, such as empirically testable predictions. Whereas demonstration 'takes place entirely within the model', interpretation is 'a function that takes us from what we have demonstrated [...] back into the world of things'. [Hughes 1996, p. 328; 333] Interpretation, thus, may be thought of as 'the inverse of denotation' (ibid.).

Applying Pincock's distinction between a physical and a mathematical attitude to the DDI account, one might say that, whereas the mathematical attitude may well be appropriate as long as one is dealing with issues of demonstration - that is, as long as one views the mathematical model 'from within' - both denotation and interpretation depend on the physical attitude, at least insofar as the latter supplies empirically meaningful content. The DDI account, thus, claims to elucidate both the general character of theoretical models and their application in specific empirical contexts. It does not, however, purport to be an exhaustive account of theoretical representation. Hughes is careful to point out that he is 'not arguing that denotation, demonstration, and interpretation [are] individually necessary and jointly sufficient for an act of theoretical representation to take place'. [Hughes 1996, p. 329] Rather, he makes 'the more modest suggestion that, if we examine a theoretical model with these three activities in mind, we shall achieve some insight into the kind of representation it provides' (ibid.). It therefore makes sense to analyse which central aspect of theoretical models each of the components brings out.

At the heart of the DDI account is the idea of denotation, which Hughes borrows from Nelson Goodman's work on representation in aesthetics, especially his Languages of Art (1976). Goodman introduces the idea in order to explain the relation between a painting and what it represents. Having dismissed the proposal that similarity determines what a painting is a picture of - after all, 'a Constable painting of Marlborough Castle is more like any other picture than it is like the Castle' [Goodman 1976, p. 5] - Goodman introduces the term 'denotation' as a placeholder for that elusive determining relation. The relation between a picture and that which it is a picture of, Goodman writes, is close to 
'the relation between a predicate and what it applies to' (ibid.). Importantly, then, denotation is conceived of as a basic relation, in that it establishes a direct connection between elements in the world and elements of a model - a connection that does not rely on problematic notions of similarity. Goodman's claim that 'denotation is the core of representation and is independent of resemblance' [Goodman 1976, p. 5] may seem to suggest that denotation automatically entails representational success, but this is not the case. (As Goodman makes clear, denotation is not a sufficient condition for representation, but at most a necessary condition for certain kinds of representation, cf. [Goodman 1976, p. 25].)

To the extent that theoretical elements denote real features of the world, the relation they bear to those features is, in a phrase endorsed by Hughes (and borrowed from Duhem), 'only the relation of sign to the thing signified' [Hughes 1996, p. 330]. Some critics have attacked the DDI account for precisely this reason; they argue that 'the notion of denotation seems to imply that the relation between model and target is purely arbitrary' [Ducheyne 2006, p. 216]. This criticism, however, misses the mark. To be sure, denotation may be arbitrary and merely stipulative, but it need not be. In the case of theoretical models, denotation will more often than not be guided by background assumptions and theoretical frameworks, as model-building is typically part of a larger project of inquiry. This naturally imposes limits on the degree to which denotation can be arbitrarily stipulated. Furthermore, the criticism overlooks that, according to the DDI account, it is the triad of denotation, demonstration, and interpretation, rather than denotation alone, which jointly establishes representation. By following Goodman's original advice that 'we must examine the characteristics of representation as a special kind of denotation' [Goodman 1976, p. 5], the DDI account does not simply equate denotation and representation. Rather, the idea is that, in order for representation to be attained, denotation must be put to the test by successful demonstration and interpretation.

Some degree of arbitrariness in denotation is not only to be expected, but may even be considered necessary for the purpose of model-building. Devising a theoretical model requires selecting the relevant characteristics of a physical system, and judgments of relevance may vary depending on explanatory context. Hence, unless one is committed to the uniqueness of theoretical models - i.e., the implausible view that, for any given physical system, there can only ever be one theoretical model that truly represents it - some freedom in choosing how to denote elements in the world is clearly desirable. This is not to say that denotation is always successful, in the sense that it always succeeds in picking out those elements which are explanatorily relevant, thereby imbuing the model with representational significance. It would indeed be miraculous if the success of denotation were guaranteed in this way. But this is not what the DDI account claims. The point is not that representation can somehow be established by mere stipulation, but rather that denotation provides a tentative, prima facie connection with the world, which may then be vindicated in the course of further demonstration and interpretation.

Of the three components of the DDI account, demonstration is perhaps the 
least problematic, since, in the case of mathematical models, it can largely be equated with the use of mathematical derivation techniques. While such techniques may have their own practical and conceptual problems, they do not directly touch upon the question of how theoretical models represent a reality external to it; demonstration, to paraphrase Hughes, takes place entirely within the model in its mathematical formulation. Nonetheless, demonstration is essential, both insofar as it allows for the derivation of results and predictions from a model, and because it makes salient that a mathematical model, by virtue of its being a mathematical object, has an internal dynamic. For Hughes, this insight is fundamental to the use of models in physics in general:

To be predictive, a science must provide representations that have a dynamic of this kind built into them. That is one reason why mathematical models are the norm in physics. Their internal dynamic is supplied, at least in part, by the deductive resources of the mathematics they employ. [Hughes 1996, p. 332]

I have argued elsewhere that 'a mathematical model may contribute new elements to the theoretical description of the physical system, or class of systems, under consideration' - elements which are extraneous to both fundamental theory and empirical data. An example would be what in mathematical physics is sometimes called 'rigorous results and relations' that may link different models in unexpected ways. It has been argued that such relations may even function as channels of empirical warrant from one model to another. [Gelfert 2009, p. 515-518.] In addition to such newly contributed elements and relations that bring out the internal dynamic of a model, there are also the considerable deductive resources contained within mature mathematical formalisms, which will be discussed in detail in the second half of this paper.

Interpretation, like the other two components of the DDI account, is constitutive of the way theoretical models represent. Without it, demonstrated results would remain merely formal results within a deductive mathematical structure, lacking empirical meaning. What is needed is 'a function that takes us from what we have demonstrated [...] back into the world of things' [Hughes 1996, p. 333], and interpretation plays this part. Whereas denotation picks out features in the world, which are then denoted by elements within a model, interpretation projects internally derived results back on to the world, where they must be assessed in terms of their empirical adequacy. There is no guarantee of success in either denotation or interpretation. However, when both are successful - when a model picks out the right features in the world, and interpretation assigns empirically correct meanings to demonstrated results - denotation and interpretation may indeed be said to be the inverse of each other, and the model as a whole may be deemed empirically adequate.

Interpretation is, of course, no simple affair, as it may require considerable ingenuity and imagination. In the case of a mathematical model, even when a result has been successfully derived within the formalism of the model equations, its empirical interpretation may not always be self-evident. As an example, consider the case of mathematical divergencies: if one or more of a model's 
variables diverge for certain parameter values, the interpreter may be faced with the choice of either dismissing it as an 'unphysical' result - for example because the corresponding physical magnitude is recognised as necessarily finite for any finite physical system under consideration - or interpreting it as an indicator of a real feature in the world (e.g., a phase transition), though perhaps one that the model is unable to capture (e.g., a phase transition). Just as in the 'inverse' case of denotation, some degree of arbitrariness is thus to be expected, perhaps even inevitable. Interpretation, however does not operate in a vacuum: just like denotation, it will be constrained by a range of commitments, presuppositions, and background assumptions. Some such constraints may be explicitly built into the model, others may simply be part of the theoretical framework that underlies the model. ${ }^{5}$ Interpretation, on the DDI account, is not merely passively presented with results which it must make sense of; rather, its operation will be informed, and at the same time constrained, by what has gone into the construction of the model up to that point.

\section{Denotational success and the role of inferential utility}

Earlier I mentioned a line of criticism according to which the DDI account renders the relation between model and target 'purely arbitrary', given that denotation carries with it an intrinsically stipulative element. It would, however, be hasty to conclude that the DDI account fails on this ground, since the DDI account construes successful representation as the joint effect of the three main ingredients, denotation, demonstration, and interpretation. That is, demonstration and interpretation may provide the DDI account with the internal resources to keep 'in check' whatever element of arbitrariness is introduced by denotation. Still, there remains the very real worry that, by making denotation the decisive element by which a model fundamentally acquires representational force ${ }^{6}$ (or directedness), the DDI account cannot exclude spurious cases, where a failure of denotation - for example, because it 'misses the target' (or targets the wrong sort of entity or phenomenon) - may go undetected during the later stages of demonstration and interpretation, simply as the result of fortuitous but non-accidental circumstances of the case at hand. If this sounds too abstract, the same point can be put more concretely, in the form of a question: Why should only denotation, not demonstration and interpretation, be involved in establishing the fundamental representational directedness of a model? After all, scientific practice appears to be replete with examples where demonstration and interpretation determine to a significant degree what a model is a model of - cases, in other words, where demonstration and interpretation constrain the direction of fit. Whether model and target stand in a

\footnotetext{
${ }^{5}$ On the notion of constraints in scientific modelling, see also Tarja Knuuttila's contribution in this volume.

${ }^{6}$ The expression 'representational force' is borrowed from (Suarez 2004).
} 
fundamentally representational relationship, is thus not a matter of denotation alone.

\subsection{Denotational indeterminacy}

As an illustration of the sort of problem that afflicts the idea of denotation as the ultimate source of representational force, let us consider the following example, which I borrow from Suárez (2004). Suppose that one wishes to represent the movement of two ships on the sea in the form of a physical model, using simple ingredients such as two pens and two pieces of paper. Intuitively, this task seems easy enough: one need only stipulate, through an act of denotation, that the paper represent the sea, and that the two pens represent the ships. Once the denotational relationship has been fixed, one can then go ahead and draw a number of (though, given the model's simplicity, perhaps not very many) correct inferences 'about the ship-on-sea system on the basis of a consideration of the pens-on-paper system, such as, for instance, that the trajectories of the ships may cross and that they may crash' [Suárez 2004, p. 772]. This much seems uncontroversial and quite straightforward, perhaps even trivial. However, given that denotation is explicitly construed as an alternative to global criteria such as similarity and isomorphy, there would appear to be no constraint that would stop us from inverting the denotational arrangement - that is, let the pieces of paper represent the ships, and the pens the sea - even though this choice would seem counterintuitive, to say the least. As Suárez observes, 'it seems so because it is less informative, since the relative movements of pens and paper can not allow us, for instance, to infer the possibility that the two ships may crash' [Suárez 2004, p. 772]. Denotation, and the multiplicity of denotational arrangements it entails, thus appear to give rise to a form of denotational indeterminacy. This suggests that whether or not a model $A$ successfully represents a state of affairs $B$ is at least in part determined by $A$ 's inferential utility, where the latter is the degree to which ' $A$ allows competent and informed agents to draw specific inferences regarding $B$ ' [Suárez 2004, p. 773]. It also suggests that, without inferential utility as a constraint on model-construction, denotation may fall foul of problems of indeterminacy.

One might worry whether the indeterminacy of denotation and the resulting ambiguity of denotational arrangements are all that significant. Surely, one might argue, 'unnatural' denotational arrangements - such as the paper's (instead of the pens') representing the ships in the pens-and-paper model - can easily be ruled out on the basis of simple criteria, such as similarity, which do not require deployment of the full inferential apparatus. While some basic recognitional capacity is needed in order to recognise a flat piece of paper as more similar to the sea's surface than a compact pointed object such as a pen, such a capacity hardly requires much in terms of the active drawing of inferences. It is, after all, what children do intuitively when they play 'battleships' using pen (or pencil marks) and paper! ${ }^{7}$ However, such a response to the worry

\footnotetext{
${ }^{7}$ Responses on the basis of psychological experience, including subjective assessments of
} 
about denotational indeterminacy would be inadequate. While it may be the case that simple physical models, such as the pens-on-paper model, do not pose serious denotational problems, the problem of denotational indeterminacy cuts much deeper. There are good reasons to think that the problem will become more pertinent as the level of abstraction increases, not least because simple criteria such as similarity quickly lose their applicability.

\subsection{Denotation and scientific realism: an example}

In order to drive home the point about the reality of worries about denotational ambiguity, let us digress briefly and consider a concrete example from physics, specifically the case of 'quasi-particles' in condensed matter physics. When modelling solid state systems (e.g., metals), one often represents the collective behaviour of correlated electrons in terms of the so-called Fermi sphere. In accordance with fundamental constraints such as the Pauli exclusion principle, correlated electrons in a solid can only occupy certain (mutually exclusive) energy states. If a certain single-particle state is fully occupied, a new electron that is added to the system must occupy the next-highest energy state that is available. In a system of many electrons, for example a solid with of the order of $10^{23}$ particles, a large number of neighbouring states will thus be occupied. Given that, in so-called reciprocal $(k-)$ space, the energy $E_{k}$ of an electron varies with the distance of a particle's state $(\vec{k}, \sigma)$ from the origin $(k=0)$, the visual model that is typically invoked is that of a sphere containing all occupied electron states, centered around the single-particle ground state $k=0$. At zero temperature, when the system occupies the many-body ground state of lowest total energy, this Fermi sphere is clearly defined, in that it has a sharply defined surface, all occupied states inside, none outside. In the presence of interactions and fluctuations, however - in other words, when the system is at finite (nonzero) temperature - the sphere will be deformed, and some electrons will be excited into states outside the original sphere, leaving behind an unoccupied state inside.

Due to the strong correlations that exist between the constituent electrons in a solid, various disturbances and excitations from the ground state can give rise to a range of collective responses of the system, perhaps the most important of which are instances of quasi-particle behaviour. Such behaviour is characterised by the (chimerical) appearance of 'new' particles, which appear to have measurable properties such as a virtual 'effective mass', average life-time, electric charge, and so forth. These so-called quasi-particles behave just like 'normal' particles across a wide range of measurements and experiments, and their effects can be reproduced experimentally in a robust and reliable manner. For example, removing an electron from the system, and with it its negative electrical charge,

inferential utility, can only go so far. As I shall suggest in the next section, a more fruitful place to look for constraints on denotational arrangements would be in their material or formal characteristics, insofar as these embody certain inferential strategies and theoretical commitments. As I will claim in Section 4, this is one of the features that make mature mathematical formalisms a powerful representational resource. 
would appear to leave an 'electron hole' behind - which may then 'act' as a positively charged quasi-particle, and as such may, for example, form bound states with other electrons (forming so-called 'excitons'). However, the existence of such quasi-particles is illusory, insofar as quasi-particles are mere artefacts of the collective behaviour of the correlated many-electron system: their apparent properties and effects do not have independent reality, but are simply functions of the total many-electron system. (See [Gelfert 2003], for a detailed argument to this effect; and [Falkenburg 2007] for a differing view.) This is most obvious in the case of an electron hole, whose causally relevant features are simply due to the absence of an electron, and not the result of the coming into existence of an independent new kind of entity; the same applies, by extension, to other quasi-particles. It must therefore be conceded that a quasi-particle, just like an absence, to paraphrase David Lewis, 'is nothing relevant at all, and therefore cannot furnish causal relata' [Lewis 2004, p. 282]. While this is not the place to explore the ramifications, metaphysical and otherwise, of 'quasi-entities' such as holes, absences, and quasi-particles, the example is nonetheless instructive with regard to the role of denotation, e.g. in ascertaining referential success. Denotation as the basis of representation has occasionally come under fire for being a success term, e.g., for positing 'a sharp difference between a representation of a real object - where the source denotes the target - and a "representation" of a fictional object, which does not involve the denotation of what is purportedly represented' [Suárez 2004, p. 770]. Indeed, to mention just one critic of denotation, Suárez regards it as a crucial advantage of a thoroughgoing inferential conception of representation that, unlike a denotation-based account, it is capable of dealing with instances of fictional representation - since fictional objects (say, the concept of a 'unicorn') can, of course, feature in inferences, even if they do not correspond to any objects in the real world.

But even if denotation cannot account for all kinds of representation, such as those involving fictional objects, it may still turn out to be a central ingredient in scientific representation. For, more often than not, denotational success is a criterion of scientific success; while it is stipulative in the first instance, it needs to prove its mettle in subsequent inquiry. The example of quasi-particle behaviour in a correlated system illustrates this point nicely: even though the collective behaviour of the system mimicks particle-like behaviour, the scientific challenge lies precisely in explaining how such emergent behaviour can arise from a system of simple constituents, without accepting a host of new (fictitious) quasi-entities as on a par with what we know, on independent grounds, to be the fundamental constituents of matter. In this particular case, at least, it would then appear that denotational success is one criterion by which to tell what is real from what is not real, whereas an inferential conception of representation, according to which 'there is absolutely no difference in kind between fictional and real-object representation - other than the existence or otherwise of the target' [Suárez 2004, p. 770] might not alone suffice to account for the comparatively higher standards of realism that apply in the case of scientific representation.

On the characterisation offered here, one of the functions of successful denotation is to furnish reference; an element $A$ of a model can only be said to 
'stand for', i.e. successfully denote, an aspect or ingredient of a physical system (or class of physical systems), if $A$ succeeds in picking out a relevant feature of reality. Matters of accuracy or completeness are of secondary importance at this denotational stage of scientific modelling, though they will inevitably become important later on - especially when one tests those (quantitative and qualitative) results that have been derived within a model, for example by comparing them against experimental results. Within the limits of what is required for referential success, denotation allows for considerable flexibility and can be multiply realised. Some critics have suggested that, while denotation may have a place in artistic representation, where 'almost anything may stand for anything else' [Goodman 1976, p. 5], it does not have a role to play in scientific models. After all, 'if the appropriate relationships are not in place between the relevant properties then the "model" will not be deemed scientific' [French 2003, p. 1478]. But this is a platitude, not a valid criticism: Of course, any attempt at representation, whether in art or science, will be subject to constraints, some of which are conventional in character, while others are due to the specific - aesthetic or cognitive - goals associated with the (artistic or scientific) representation in question. In the case of scientific representation, denotation needs to be constrained by demonstration and interpretation (as the DDI account suggests), as well as by other constraints (for example, the commitment to the referential success of central elements of a model, as in the quasi-particle case above). Given that scientific models serve cognitive goals, inferential utility, too, can act as a constraint on model-based representation. In other words, one should be careful not to overstate the antagonism that exists between denotation-based accounts of scientific representation on the one hand, and inferential accounts on the other hand. Both have their place in accounting for scientific representation, and may even be complementary. In much the same way that mere denotation may not be enough to establish representational force, one might argue that inferential utility alone, without a denotational basis, may not suffice for the success of a model. A successful scientific model, I want to submit, is one that allows us to draw inferences and gain information about its target system, where the latter may be a specific physical system or itself a (generalised) class of phenomena.

\subsection{The heterogeneous character of scientific representa- tion}

What inferential and denotational accounts have in common is their acknowledgment that model-based representation depends for its success on a variety of factors - such as the cognitive goals of model-users, referential success, and interpretation - which cannot easily be assimilated, e.g., to a purely structuralist account based on isomorphism. Structuralist accounts may well be adequate for a suitably narrow class of scientific models, perhaps comprising only those kinds of models where 'messy' problems of interpretation and denotational indeterminacy no longer arise. In his discussion of the DDI account, Steven French asks: 'One must also include some account of interpretation, but if one is going to do that, why bother with denotation to begin with? Why not return to an 
account based on isomorphism?' [French 2003, p. 1479] Similarly, in a footnote to the same paper, French refers to work by Hendry and Psillos in which they develop an 'interactive view' [Hendry 1999], according to which theories and models combine elements from different representational media: 'On such a view, representation might be analyzed in terms of some combination of denotation and isomorphism although it is not clear quite how this would work.' [French 2003, p. 1478] Such rhetorical questions and quick dismissals, however, only carry weight if one is already operating on the assumption that denotation, interpretation and, by extension, considerations of inferential utility - in spite of their being conceptually distinct from isomorphism - are incapable of giving rise to any interesting questions of their own. What needs to be realised, however, and what has perhaps not yet been adequately acknowledged, is that the multiple accounts of representation discussed in the present paper can be thought of - much like Hendry and Psillos's 'interactive view' - as attempts to make sense of representation as a heterogeneous phenomenon. The mere fact that this is an ongoing project which does not argue towards a fixed conclusion and still needs to address many open questions, should not be held against it; such is the nature of live philosophical projects. The real challenge, then, consists not in concentrating on cases where one of the candidate sources of representational force - where it be denotation, inferential utility, or any of the other possible sources - fails, but in investigating the interplay between them.

Recognising that successful representation is typically the joint effect of an interplay of multiple factors, is necessary, not least in order to account for the diversity of actual scientific models. Most philosophical discussions of scientific models - this paper included - tend to focus on theoretical or mathematical models. However, just as it would be hasty to dismiss examples that cannot be assimilated to one's preferred (e.g., structuralist) account of representation, so it would be careless to dismiss a whole tradition of philosophical thinking about mechanical and material models, simply on the ground that such models are specified directly in their materials, rather than in their relation to theory. The denotational and inferential accounts of representation can be usefully applied to such material models, as has recently been argued by Davis Baird (2004). Baird discusses both the example of orreries - devices which mechanically represent the motion of various planets and moons - and Watson and Crick's (physical) model of the DNA double helix. In those cases it is entirely appropriate to say that 'the sticks in Watson and Crick's [double-helix] model denote bond lengths, not rigid metallic connections', and that one can use an orrery - in a hands-on way, by setting it in motion - 'to demonstrate [...] the shape of the moon's orbit relative to the sun'. [Baird 2004, p. 38; my italics.] ${ }^{8}$ As James Watson reports in his well-known first-person account, The Double Helix (1968), manipulating the physical model became not just a convenient shortcut for theoretical reasoning based on empirical data, but an independent source of inferential utility:

\footnotetext{
${ }^{8}$ It is again telling that some structuralist critics relegate denotation to, at best, the semantic realm of 'how words represent' [French 2003: 1478] - i.e., of linguistic meaning - thereby ignoring the manifestly denotational aspects of some of our non-linguistic ways of interacting with the world.
} 
'The $\alpha$-helix had not been found by only staring at X-ray pictures; the essential trick, instead, was to ask which atoms like to sit next to each other. In place of pencil and paper, the main working tools were a set of molecular models superficially resembling the toys of preschool children.' [Watson 1981, p. 34] From the philosophical armchair, tactile manipulation of a physical model may look like a poor substitute for theoretical derivation, but it may acquire paramount importance 'when conceptual manipulations are impossible either for lack of a theory or because analytical manipulations would be too difficult' [Baird 2004, p. 39]. Either way, material models as well as theoretical models allow their user to articulate and infer knowledge of the part of the world they represent; successful denotation is an important component in this relation, as is inferential utility, which allows for the extraction of information about the target system.

\section{Mathematical formalism as interpretative re- source and inferential practice}

Let us step back for a moment and take stock. In the preceding sections, I argued that representation needs to be understood as a heterogeneous phenomenon, which, except in trivial cases, is brought about by the presence of more than one source of representational force. I pointed to several recent proposals in support of the thesis that no single source - denotation, inferential utility, let alone similarity - is enough to guarantee representation. Hughes' DDI account goes some way towards acknowledging that representation is achieved through the interplay between three different theoretical activities. Pincock's division of the modelling process into a sequence of matching, derivation, and application models, along with his distinction between a 'physical' and a 'mathematical' attitude, likewise recognises that different theoretical attitudes and activities need to combine to give rise to successful representation. That representation cannot be achieved by stipulation alone, but needs to be both underwritten by referential success (at least of the most central ingredients of a model) and informed by considerations of inferential utility, was demonstrated by examples of what I had tentatively referred to as 'denotational indeterminacy'.

Of those who acknowledge - at least implicitly - the heterogeneous nature of representation, Suárez goes perhaps the farthest: In the course of defending a deflationary account of scientific representation, he argues that, in light of our failure to come up with universal necessary and sufficient conditions for representation, we should conclude that there are 'no deeper feature to scientific representation other than its surface features' [Suárez 2004, p. 769] - where the dominant 'surface feature' of a representational model is its inferential utility as a source of 'surrogate reasoning'. This inferential conception of scientific representation purports to be at the same time deflationary and pragmatic: It is deflationary, insofar as it only aims to identify minimal requirements for representation, without assuming that these must take the form of necessary and sufficient conditions, let alone be formulated in terms of isomorphism and 
similarity (see also [Suárez 2003]); and it is pragmatic, insofar as inferential utility varies across groups of 'competent and informed agents', whose ability to draw valid inferences regarding $B$ on the basis of reasoning about $A$ is itself 'a pragmatic skill that depends on the aim and context of the particular inquiry' [Suárez 2004, p. 773]. Suárez makes a convincing case that a universal 'onesize-fits-all' account of scientific representation is likely to be an illusion, and limits himself to identifying at most two general necessary conditions - representational force and inferential utility - that need to be in place for scientific representation. What generates representation in each successful case, beyond the mere fulfillment of these two necessary conditions, is not something that can be prejudged by abstract analysis: 'In every specific context of inquiry, given a putative target and source, some stronger condition will typically be met; but which one specifically will vary from case to case.' [Suárez 2004, p. 776] While I concur with Suárez that the search for a substantial account of scientific representation - that is, an account in terms of universal necessary and sufficient conditions for scientific representation - is unlikely to succeed, I believe that many positive instances of representational success can nonetheless be explained, in a unified way, by identifying, across a range of specific cases, what it takes to achieve representation. ${ }^{9}$ What gives unity to model-based representation as a successful scientific practice, I suggest, is not an abstract requirement (e.g., the presence of isomorphism, similarity, interpretation, etc.), but instead is a matter of general features of the process of model construction. In the remainder of this paper, I shall argue that, more often than not, the deployment of a mature mathematical formalism during the process of model construction and articulation, plays an important role in achieving the kind and degree of unification required for scientific representation.

\subsection{Hesse on mathematical formalism}

As the starting point of my defence of mathematical formalisms as a unificatory force, I shall draw on some of the early work on the philosophy of scientific models, in particular the work by Mary Hesse. While Hesse is best-known for her view of models as analogies [Hesse 1963], much of her work also contributes to an analysis of the role of mathematics in physics. Thus, in her 1953 paper 'Models in Physics', Hesse sets out to defend two theses, the second of which is the suggestion that 'most physicists do not regard models as literal descriptions of nature, but as standing in a relation of analogy to nature' [Hesse 1953, p. 201; emphasis original]. It is her detailed defence of this second claim that sparked a long-lasting debate over the analogical character of models. Much less has been made of Hesse's first claim, however, which asserts that 'mathematical formalisms, when used as hypotheses in the description of physical phenomena, may function like the mechanical models of an earlier stage in physics,

\footnotetext{
${ }^{9}$ This difference is partly a matter of whether one wishes to emphasise the role played by specific features of a given model, or whether one is more optimistic about the possibility of identifying general features that generate successful representation for a whole class of models. (See also [Suarez 2010, p. 98].)
} 
without having in themselves any mechanical or other physical interpretations' [Hesse 1953, p. 199; emphasis original]. It is this claim - which points to the significance of extant mathematical formalisms in physics - that I wish to focus on in the remainder of this paper. In particular, I shall argue that the existence of mature mathematical formalisms often plays an important role in bringing about successful representation by mathematical models. In order to make this case, I shall have more to say about what the term 'mathematical formalism' is meant to capture, and how Hesse's suggestion can be adapted to the contemporary debate. Before delivering on this promise, however, I shall try to unpack Hesse's position in a little more detail.

Hesse's early work must be understood against the backdrop of a tradition that largely equated scientific models with either material or mechanical models. On this view, which derives from 19th-century scientific usage, a typical scientific model is 'a (real or imagined) concrete, material representation of something' [De Regt 2005, p. 215]. Obvious examples would include the billiard-ball model of an ideal gas, or Maxwell's vortex model of the ether. By the time Hesse embarks on her analogies of models and analogies, the term 'model' has proliferated beyond the realm of the mechanical or 'picturable' to also include, for example, quantum-mechanical models. Indeed, it is this proliferation of the use of the term 'model' - expressed in the claim that a model can be 'any system, whether buildable, picturable, imaginable, or none of these' [Hesse 1963, p. 21]- which motivates Hesse's project. Her indebtedness to the traditional view of models as concrete or picturable 'stand-ins' for real systems, however, does shine through in her characterisation of the role of mathematical formalism in many of the newer scientific models:

The question then arises, what takes the place in these physical theories of the pointers towards further progress which are provided by an easily pictured mechanical model? I shall suggest that what takes their place is provided by the nature of the mathematical formalism itself - any particular piece of mathematics has its own ways of suggesting modification and generalisation; it is not an isolated collection of equations having no relation to anything else, but is a recognisable part of the whole structure of abstract mathematics, and this is true whether the symbols employed have any concrete physical interpretation or not. [Hesse 1953, p. 200]

While I shall subsequently move beyond Hesse's account of mathematical formalism, I here wish to point to three elements of the above quote that strike me as especially instructive. First, Hesse credits mathematical formalism with providing 'pointers towards further progress', similar to the way picturable mechanical models in the past have suggested new avenues of research to those who devised them. The tentative language is important here: picturable mechanical models as well as mathematical formalism point towards progress and suggest new steps of modification and generalisation, rather than making progress inevitable or entailing specific modifications. At the same time - this is the second 
point, which is at least implicitly present in the quote from Hesse - mathematical formalism not merely accommodates whatever hypotheses one may already have about the system in question, but instead also constrains the ways in which a system can be represented. As a 'particular piece of mathematics' each formalism 'has its own ways of suggesting modification and generalisation' (ibid.; my emphasis); some modifications may go beyond, or even 'violate', the constraints that hold for a given formalism, without thereby being logically or mathematically non-permissible. ${ }^{10}$ Note also that a mathematical formalism need not be identifiable with any particular branch of mathematics as such; 'formalisms', in the sense discussed here, will typically be more local than any branch of mathematics such as topology, number theory, algebra, etc. For the same reason, not just any application of mathematics to nature will count as employing a mathematical formalism; as Hesse indicates, a formalism will be 'a recognisable part of the whole structure of abstract mathematics', not the totality of mathematics itself. ${ }^{11}$ Finally, as a third observation in connection with Hesse's quote, I wish to emphasise the pragmatic dimension of mathematical formalism. A piece of mathematics, such as an interpreted set of equations in conjunction with rules and heuristics for their application, must be recognisable as such by the user; its value lies in its utility to the user, for example in the extent to which it enables the user to derive empirical results or to pursue those modifications and generalisations that Hesse identifies as 'pointers towards further progress'.

\subsection{Mathematical formalisms vs. mathematical techniques and formal frameworks}

In order to gain a better understanding of the significance of mathematical formalisms in model-based science, I shall contrast what I take to be three main heuristic uses of mathematics in scientific inquiry. Each of the three has its own distinctive way of shaping the future course of inquiry, either by shaping its immediate goals or by constraining its possible outcomes. While there may be some borderline cases that cannot be classified unambiguously, I believe that, in most cases of actual scientific practice, it will be sufficiently clear which of the three categories an instance of applying mathematics belongs to. As labels for these categories, I suggest a trichotomy consisting of the terms 'mathematical techniques', 'formal frameworks', and 'mathematical formalisms'. I shall briefly describe each category, characterise its role in scientific inquiry, and illustrate its significance on the basis of an example.

Scientists often use the expression 'mathematical techniques' as an umbrella term for a wide variety of mathematical approaches. A quick database search for the term 'mathematical techniques' returns the following (needless to say: incomplete) list of examples: 'the Fourier transform', 'regression analysis', 'cal-

\footnotetext{
${ }^{10}$ This will become clearer once I discuss examples of extant mathematical formalisms in the next subsection.

${ }^{11}$ Hence, when I speak of the 'unificatory power of mathematical formalism', as I shall later in this section, I do not intend this to be synonymous with Eugene Wigner's phrase of the 'unreasonableness effectiveness of mathematics in the natural sciences'.
} 
culus', 'partial differential equations', 'method of least squares', 'Green's function techniques', 'discretization', and so forth. Clearly, then, actual scientific usage is of limited usefulness in assigning a precise meaning to the term. However, even though the individual items on this (or any similar) list may not seem to have much in common with one another, the investigative contexts in which scientists help themselves to such 'mathematical techniques' nonetheless share certain general characteristics. Typically, and by no means trivially, references to 'mathematical techniques' usually occur in contexts where the generation of (often numerical) results is the main concern. Thus, mathematical techniques may be employed to reduce complexity, predict numerical outcomes, extract data, or calculate expectation values. What counts as a 'result' - that is: as an outcome that will bring inquiry, at least momentarily, to an end - depends on the overall goal of the investigator: for a mathematical physicist, being able to write down a solution in a mathematically closed form might suffice, whereas a computational physicist might not be content until a numerical value (perhaps within a certain margin of error) has been calculated. ${ }^{12}$

For the purpose of the present argument, I shall understand by 'mathematical techniques' those outcome-oriented uses of mathematics that aim at generating empirically adequate results, either numerically or derived through approximations, which may or may not be considered ad hoc by the lights of fundamental theory. With mathematical techniques, given the wide range of approximations and methods employed across the sciences, it is difficult to single out any one example in particular. Most approximation techniques for systems of differential and integral equations (including discretization and finite element methods) would fall into this category, as would variational methods which, in a purely formal way, map analytically intractable problems onto optimization problems that may then be solved numerically. It is not untypical, within the class of 'mathematical techniques', to find a lot of continuity between techniques for deriving results and methods of data analysis. Thus, techniques such as applied factor analysis may be understood equally as '[1] a tool for uncovering the order, patterns or regularity in data or [2] as a mathematical mold of casting scientific theory' [Rummel 1970, p. 12]. Indeed, one could argue that it is in the nature of the 'outcome-orientedness' of mathematical techniques, as defined here, that little distinction is made between results that have been derived from fundamental theory, and data that have been obtained otherwise, since ideally there should be a perfect fit between them.

There is a stark contrast between the application of mathematical techniques with the purpose of generating results and the use of mathematics as a provider of what I shall call a formal framework. Unlike in the case of 'mathematical techniques', scientists do not themselves typically use the term 'formal (or mathematical) framework'; hence, whereas in the former case, it was necessary to 'clean up' scientific usage in order to narrow down the meaning of the term, in the case of the notion of 'formal framework' some degree of 'extrapolation'

\footnotetext{
${ }^{12}$ Tarja Knuuttila, in her contribution to this volume, links the 'results-orientedness' of much of scientific modelling to the 'concrete manipulability' of models.
} 
will be necessary in order to make clear what element of scientific practice the term is supposed to capture. To this end, it will be useful to begin by drawing a few contrasts. Whereas mathematical techniques are typically tailored to specific empirical contexts, formal frameworks are usually established in a more 'global' way, without much regard for the details of any particular empirical situation. Also, whereas the choice of mathematical techniques often takes place well into the process of inquiry - for example, when it turns out that a derivation from 'first principles' is too complex and that numerical approximations need to be made - the choice of a formal framework often precedes the formulation of more specific problems, which may subsequently be tackled. Finally, whereas mathematical techniques are usually evaluated in terms of their usefulness for reproducing empirically adequate results, formal frameworks typically have a more strongly normative flavour, in the sense that they are either underwritten by general theoretical commitments (though not necessarily derivable from fundamental theory) or may even be constitutive of specific theoretical research programmes. A good example of the use of mathematics for the purpose of establishing formal frameworks is the significance, within certain areas of fundamental physics, that has been (and continues to be) attached to considerations of symmetry. Mathematical symmetries are, of course, an integral part of many fundamental laws of contemporary physics, so it may seem that arguments from symmetry constitute simply an appeal to fundamental theory rather than an instance of imposing a (mathematical) formal framework. However, if one looks at the history of modern physics, it becomes clear that in crucial periods of, for example, the evolution of modern quantum theory, group theory i.e., the mathematical theory of symmetry - provided a formal framework for the development of physical theory itself.

The example of group theory as a formal framework for the formulation of quantum mechanical problems deserves some more attention. An important historical role is played by the mathematician Hermann Weyl. (For an insightful discussion of the relation between mathematics in physics in Weyl's work, see [French 1999].) In his monograph The Theory of Groups and Quantum Mechanics (first published in German in 1928), Weyl provides what is perhaps best described as an 'embedding' of the nascent quantum theory of the atom into the mathematical framework of group theory. Many of the complex phenomena found in atomic physics - such as the finer details of atomic spectra - were thought to be analytically (and computationally) intractable using the by then established formalism of the Schrödinger equation. Starting from the heuristic recognition that, when it comes to systems of several electrons interacting with one another as well as with the nucleus of an atom, 'nothing is known on a purely theoretical basis (apart from a rough estimate of their positions) except their symmetry properties' [Weyl 1928, p. 181], Weyl proceeded to give an account in terms of symmetry properties 'independently of the dynamical structure of the physical system under consideration' [Weyl 1968, p. 276]. The very fact that this approach was neither tailored to specific empirical cases nor directly entailed by the known dynamical laws of quantum theory, came to be seen as an advantage: Group theory, according to Weyl, revealed 'the essential 
features which are not contingent on a special form of the dynamical laws nor on special considerations concerning the forces involved' [Weyl 1928, p. xxi]. Its very independence from - perhaps even disregard for - specific empirical detail, along with an at best loose relationship to what was known about fundamental theory at the time, allowed group theory to function as a formal framework for atomic physics and, later, nuclear physics, where the pursuit of symmetry relations (for example via the standard model of elementary particles) became a guiding principle of theorising. ${ }^{13}$

The two uses of mathematics distinguished thus far - as, on the one hand, a set of techniques for the 'local' generation of specific results and, on the other hand, a formal framework for physical theorising - define different ends of a spectrum of deploying mathematics in scientific inquiry. I now wish to turn to a third mode of using mathematics in science, which occupies a middle position between the two extremes outlined above, but which at the same time has characteristic features that cannot be reduced to an admixture of elements from either mathematical techniques or formal frameworks. I shall refer to this use of mathematics in science as the deployment of a mathematical formalism. As with the two previous cases, some care must be taken to give a specific meaning to the term 'mathematical formalism'. 'Mathematical formalism', in the present context, is to be understood as a physically interpreted formalism, i.e. as more than merely the deployment of the formal language of mathematics. It must be sufficiently general to be applicable to a range of physical problems; at the same time, it will typically fall short of universality, in the sense that certain cases - though logically and physically permissible - nonetheless do not lend themselves to the application of the formalism. It thus follows that a mathematical formalism will typically include rules and heuristic assumptions that are not themselves part of either the mathematical framework or entailed by the 'underlying' fundamental theory.

The general features mentioned so far are inferred from the assumption that mathematical formalisms inhabit a middle ground between outcome-oriented mathematical techniques and formal mathematical frameworks. What needs to be shown is that such a middle ground does indeed exist, and this can be done by way of example. In the remainder of this subsection, I wish to discuss in some detail what I take to be a good example of a mature mathematical formalism in physics, in the sense that has been discussed so far. The example I have in mind is the formalism of creation and annihilation operators in quantum manybody physics. I shall first give a theoretical justification (or rather: motivation) for this formalism, before giving examples of how it is deployed in practice. However, it is worth emphasising beforehand that the mere possibility of giving a theoretical justification for a given formalism does not entail that any particular application of that formalism is best understood as a 'direct' application of fundamental theory to a specific problem. A mature formalism will typically be set up in such a way that its output will 'automatically' satisfy certain

\footnotetext{
${ }^{13}$ This applies to the Standard Model of particle physics as well as its proposed supersymmetric extensions.
} 
theoretical desiderata; at the same time, there may be certain theoretically permissible situations or scenarios that cannot be adequately represented using the formalism in question. A formalism, one might say in a turn of phrase that has usually been reserved for scientific models, mediates between theoretical 'first principles' and descriptions of empirical situations in their full complexity.

With this caveat in mind, I shall turn to a description of the formalism of creation and annihilation operators and their place in the quantum physics of many-body systems. While the formalism of second quantization is typically associated with quantum field theory, for example in high-energy physics, its analogue in the case of quantum many-body systems has received far less attention in the philosophical literature. The use of creation and annihilation operators is an attempt to extend the formalism of second quantization to strongly correlated many-body systems, such as delocalised electrons in a metal. In such a situation, the electrons can be thought of - to a (very crude) first approximation - as being uniformly spread-out across the crystal lattice of the solid. It is then possible to analyse the behaviour of this uniform 'sea' of electrons in terms of a superposition of 'electron waves', each characterised by a unique wave vector $\vec{k}$ (as well as a spin variable $\sigma$ ). One may then describe changes in the system, for example an energy excitation caused by a perturbation of the system, in terms of the creation and annihilation of new electron waves - adding and removing contributions to the superposition. In quantum mechanics, these processes are described by operators acting on the many-body quantum state of the system. It is these operators that, for obvious reasons, are known as creation and annihilation operators, and are symbolized by $\hat{a}_{\vec{k}, \sigma}^{\dagger}$ and $\hat{\alpha}_{\vec{k}, \sigma}$, respectively. This mathematical description of the different components of the electron system stresses the collective nature of its dynamics. ${ }^{14}$ As representations of fermions (spin-1/2 particles such as electrons), the operators must satisfy certain anticommutation relations in order to satisfy the basic constraints of fundamental theory.

So far, I have only indicated which mathematical framework - in this case, operator algebra - the formalism draws on, and how its symbolic conventions are to be understood. However, for a physically interpreted mathematical formalism to emerge, it is necessary that certain theoretical, or other (e.g., methodological), commitments are embodied in the rules and criteria for what constitutes a meaningful application of the formalism. As I have already indicated, mathematical formalisms occupy a middle ground between global mathematical frameworks and specific local applications of mathematical techniques; their usefulness to, for example, a scientist interested in modelling a specific class of physical systems, consists in narrowing down the wide range of what is logically or physically permissible (as judged from fundamental theory), without thereby precluding the application of the same formalism to different specific empirical situations.

\footnotetext{
${ }^{14}$ Thanks to the regularity of the crystal lattice, however, one can perform a (discrete) Fourier transformation, which maps the representation back onto individual electrons associated with lattice sites. Hence, even though the electrons are delocalised, we may nonetheless speak of the creation $\left(\hat{a}_{i, \sigma}^{\dagger}\right)$ and annihilation $\left(\hat{a}_{i, \sigma}\right)$ of an electron of spin $\sigma$ associated with lattice site $i$.
} 
It is crucial, then, that a formalism provides both additional constraints and sufficient flexibility to allow for its application to different target systems. By constraining the process of inquiry without tailoring it to any one empirical situation in particular, the choice of mathematical formalism may itself play an important heuristic role, for example in strategies of model-building.

As an example of the constraining role of mathematical formalism, I wish to discuss the case of the conservation of particle number in quantum many-body systems. Condensed matter physicists know on independent grounds that, in the systems they are trying to model, particles (=electrons) cannot simply be annihilated completely or created out of nothing (at least not by the mechanisms that govern the dynamics in a solid at room temperature). However, nothing in the mathematics of operator algebra per se rules out the possibility of varying particle number. Indeed, if one looked in isolation at how creation and annihilation operators are mathematically defined, then, on the standard interpretation of quantum operators, their net effect would be such that, considered individually, they would seem to describe states where an individual electron has been created from nothing, or destroyed without trace, thereby violating conservation of particle number. It is, of course, clear that any mathematical approach that could not account for the fact of particle conservation at low energies would be of little representational value for the description of real, existing many-body systems. However, the mathematical formalism of creation and annihilation operators goes beyond the mere mathematical definition of the operators themselves, in that it also includes heuristic rules for the deployment of such operators. One such rule states that creation and annihilation operators can never occur individually in the equations of our mathematical model, but only in pairs. This way, an electron that is 'annihilated' at one point in the system will be simultaneously 'recreated' at a different point in the system, and at no point is there a violation of particle number. Because the mathematical formalism does not include a variable for time, there is no 'time lag' (so to speak) between the removal of an electron at one place and the addition of another electron at a different place in the system. Despite of the lack of a temporal dimension, physicists nonetheless choose to give a dynamic interpretation to the effect of the joint application of an annihilation and a creation operator (never mind the absence of a dynamic component from the model): they claim - perhaps: pretend - that what is 'being modelled' is the movement of an electron from the place where it was 'annihilated' to the place where it was (re-)'created'.

I have here given only the briefest sketch of the example of creation and annihilation operators in many-body physics. What a closer analysis would show is that the corresponding mathematical formalism is the combination of a mathematical framework (operator algebra) with a fundamental physical theory (quantum mechanics), in conjunction with additional assumptions and heuristic rules that are neither entailed by fundamental theory nor tailored to any specific empirical problem in particular. Such substantive assumptions and constraints - including whatever theoretical justification it might be possible to give for them - are not always explicitly stated, but are often implicit, for example in 
the rules for manipulating symbolic representations of the mathematical objects in question. For creation and annihilation operators, certain rules apply concerning which combinations of operators can be substituted for others, most obviously in the case of the anti-commutation relations. At the same time, as is obvious in the example of how creation and annihilation operators are deployed in order to model 'dynamic' movement of electrons in a solid, mature mathematical formalism in physics also allow for a considerable degree of interpretative flexibility. It is for this reason, I want to suggest, that mathematical formalisms play a significant role in the construction of scientific models.

\subsection{Two contrast cases: 'mathematical moulding' and 'the structuralist approach'}

The specific character of mathematical formalisms, as outlined in the preceding two subsections, can be delineated further by contrasting it with two related, yet different, philosophical conceptions of the role of mathematics in modelling. Following their main proponents, I shall call these alternative conceptions 'mathematical moulding' [Boumans 1999, p. 90f.] and the 'structuralist' (or mapping) approach (see [da Costa and French 2003] and references therein). It needs to be emphasised that, by drawing contrasts between these three accounts of the role of mathematics in science, I am not suggesting that they are necessarily mutually exclusive. Rather, each emphasises different formal aspects of the mathematics employed, and each is characterised by qualitative differences between the kinds of theoretical activities involved in achieving model-based representation - i.e., between mathematical moulding, the mapping of structures, and the deployment of mathematical formalisms. In particular, I hope to show that mathematical formalisms occupy an interesting middle ground between more 'local' cases of mathematical moulding and the more 'global' picture sketched by the structuralist approach.

The term 'mathematical moulding' is due to Marcel Boumans, who, in the context of analysing business-cycle models in economics, defines it as follows:

Mathematical moulding is shaping the ingredients [of a model] in such a mathematical form that integration is possible, and contains two dominant elements. The first element is moulding the ingredient of mathematical formalism in such a way that it allows the other elements to be integrated. The second element is calibration, the choice of the parameter values, again for the purpose of integrating all the ingredients. [Boumans 1999, p. 90]

As Boumans sees it, model-builders in economics are typically confronted with a diversity of 'ingredients', which include 'theoretical ideas, policy views, mathematisations of the [business] cycle, metaphors and empirical facts'. The modellers' main goal 'in the context of discovery is the successful integration of those items that satisfy the criteria of adequacy' [Boumans 1999, p. 67]. In such contexts of modelling, 'mathematical moulding plays a specific role', namely: to match a model to a specific empirical situation. Model-builders typically pursue 
'an adaptive strategy until all ingredients [are] integrated'; often, one finds that 'in the integration process, "tuning" is essential': This fine-tuning 'is essential to ensure that the mathematical representation has empirical significance'. In the particular case study, an essential part of mathematical moulding consist in the models' parameter values being 'chosen such that the model could precisely mimic specific facts about the cycle' [Boumans 2005, p. 50].

The idea that models need to be 'moulded' to fit specific empirical contexts coheres well with other approaches that draw their inspiration from case studies of actual scientific practice. Thus Margaret Morrison, in a contribution to the same volume that contains Boumans' 1999 paper, argues that 'model construction involves a complex activity of integration' [Morrison 1999, p. 44]; like Boumans, she elsewhere emphasises that 'the proof or legitimacy of the representation arises as a result of the model's performance in experimental, engineering and other kinds of interventionist contexts' [Morrison 1998, p. 81]. A similar sentiment is expressed by Suárez when he writes that models are 'inherently intended for specific phenomena' [Suárez 1999, p. 75]. What these remarks indicate is that models are frequently employed in specific (often explanatory) contexts, when a phenomenon cannot be adequately described, or perhaps cannot even qualitatively accounted for, by (limiting cases of) fundamental theory. Hence, phenomenological models come to be seen as the rule, rather than the exception, across much of science. However, while this shift in perspective, from models as an intermediate step in applying theory to the world to models as representations of specific phenomena, has generally sharpened an awareness of how models are being used in actual scientific practice, I believe it does not adequately capture the role of mathematics in achieving representation. 'Mathematical moulding', with its goal of enforcing empirical adequacy of a model by fine-tuning its ingredients, appears to put too much emphasis on the empirical performance of a model in a specific situation. The second element identified by Boumans - calibration as 'the choice of the parameter values' conveys the connotation of 'curve-fitting', which many model-builders seek to avoid. Indeed, integrating 'all the ingredients' in many cases of scientific modelling may neither be feasible nor desirable; much model-building is motivated by the recognition that the 'adding of details with the goal of improving $[\ldots$ the] model is self-defeating - such improvement is illusory' [Batterman 2002, p. 22]. This is particularly salient in the case of what Robert Batterman calls 'minimal models', which are meant to shed light on the universality of certain phenomena, such as the near-identical behaviour of various physical systems in the vicinity of a critical point of a phase transition. What is needed, then, is an elaboration not only of how mathematics provides ways of fitting a given model to a specific empirical situation, but also of how it enables some models perhaps even the majority of mathematical models - to represent an entire class of phenomena, and sometimes different such classes altogether. Mathematical formalisms are an obvious candidate for providing the requisite balance between specificity and generality.

Let us turn to the second contrast case, which, for want of a better designation, I shall refer to as 'the structuralist approach'. I here have in mind a cluster 
of proposals, all of which attempt to make sense of the role of mathematics in physics, and of the relation between theories and the kinds of models actually used by scientists, in terms of an embedding of an - already mathematised theory $T$ into a mathematical structure $M$. This embedding is construed in settheoretic terms, by positing the existence of a suitable homomorphism between $T$ and $M$. Typically, according to the structuralist proposal, this will take the form of an isomorphism that maps $T$ onto a substructure $\mu$ of $M$. Introducing a formal calculus, on which $T$ and $\mu$ are taken to be isomorphic models, and assuming a way to patch this calculus up with the semantic resources for $M$, then allows one to properly regard $T$ as embedded into a larger structure most importantly, one that contains 'surplus structure' (given that $M$ is to be understood as an extension of $\mu$ ). [Redhead 1975, p. 87] What renders this picture attractive, for the present argument, is its ability to make plausible how mathematics - more specifically: the 'surplus' mathematical structure that is made available by embedding a theoretical structure into a larger mathematical framework - may be of heuristic value, insofar as new mathematical resources become available that may eventually suggest new theoretical developments. The structuralist approach has since been extended and refined, most recently through the influential 'partial structures' programme (for a review and defence, see [da Costa and French 2003]). Such refinements are partly a response to claims that a structuralist account (of the general kind sketched above) is unable to account for the diversity of the kinds of models - including analogies, iconic models, material models - that scientists actually deploy in their everyday work. One move has been to relax some of the requirements of earlier structuralist accounts, for example by demanding only partial identity between models and situations and by allowing partial isomorphisms to take the place of full isomorphisms. Some critics, however, have argued that, while partial structures and partial isomorphisms bring greater flexibility, they also are in 'danger of trivializing our representational relationships' [Pincock 2005b, p. 1254]; in response, proponents of the partial structures approach have suggested that it may be possible to appeal to heuristic factors in order to explain why one model (or one partial isomorphism) rather than another was adopted. This is not the place for a detailed discussion of the overall merits of either the 'partial structures' approach or the criticism that has been levelled against the structuralist approach more generally; instead, I want to argue that, irrespective of whether such an approach can be successfully defended, relations between abstract structures do not exhaust the role of mathematics in the construction of scientific models.

Even if one grants that there are no in-principle obstacles to giving a reconstruction of models and theories in terms of relations between abstract structures, such an approach misses an important dimension of the model-building process. The 'hands-on' character of applying a mature mathematical formalism in order to represent phenomena and physical systems seems strangely absent from the world of relations between abstract structures. ${ }^{15}$ Mathematical for-

\footnotetext{
${ }^{15}$ This need not indicate a lack of interest in scientific practice generally, since within the
} 
malisms and equations, though no doubt part of 'mathematics-at-large', are actively used to predict, explain, and represent the behaviour of real physical systems - mathematical modelling as an activity points outward to the world. Robin Hendry and Stathis Psillos put this point nicely, if mildly polemically, when they issue the following recommendation:

We should not be tempted to reify structure as something attributed in our descriptions: to consider a thing under an abstract (or structural) description is neither to think of it as an abstract object, nor to think of it as something that bears a structural relation to one. Embedded in theories, mathematical equations can be used to make sophisticated and abstract claims about real physical systems; the representational cash value of mathematics, within science, must lie in the truth-conditions of the claims it can be used to make about them. [Hendry and Psillos 2007, p. 145].

While I shall not take issue with the structuralist approach as far as it goes, I believe that the quote from Hendry and Psillos conveys a justified sense of dissatisfaction, insofar as the focus on mere structures and their relations obscures the value of mathematics as something that is used, in specific ways that are underdetermined by any structuralist account, in order to represent specific physical phenomena and (classes of) physical systems. Mature mathematical formalisms in physics, by contrast, typically include (often heuristic) rules and strategies for modelling relevant aspects of phenomena and systems that fall within the domain for which the formalism has been devised.

As I suggested at the beginning of this section, my goal in considering mathematical moulding and the structuralist approach as contrast cases to mathematical formalism was not to argue for any incompatibility between the latter and either of the former two. Rather, I intended to situate mathematical formalism - as an integral part of model-building - between the two. For, as I hope to have made plausible, mathematical moulding and the structuralist approach lie at opposite ends of a whole spectrum of conceptions of how mathematics applies to the world. Loosely speaking, whereas mathematical moulding accounts for the local fitting, or 'fine-tuning', of a model to specific empirical contexts, the structuralist account takes a global account of how theories and models can be embedded in 'mathematics-at-large', without necessarily much concern for specific features of a given model beyond its 'embeddability', as it were. Both the local and the global roles of mathematics are important and interesting in their own right. At the same time, there is ample room for intermediate positions between the two ends of the spectrum defined by mathematical moulding and the structuralist approach; it is this middle ground, I am suggesting, that is occupied by mature mathematical formalisms in science. ${ }^{16}$

structuralist approach there has been a fair bit of discussion of the history of science, especially in relation to theory change. Rather, what this indicates is that intertheory relations lend themselves more easily to subsumption under a structuralist framework than does the activity of model-building.

${ }^{16}$ In his book How Economists Model The World Into Numbers, Boumans hints at a similar 


\subsection{Mathematical formalisms as inferential and interpre- tative resources}

On the view I am putting forward, when a mature mathematical formalism is being deployed in a certain area of science, it already comes imbued with certain background assumptions regarding its application and interpretation. For example, in the case of the creation and annihilation operators discussed above, there are what one might call clear 'guidelines' as to which combinations of operators are admissible as potential representations of occurrent processes in the many-body system to be described. Certain combinations of operators can be immediately ruled out as 'unphysical', simply on the basis that they can be seen to violate basic constraints, such as conservation of particle number, which are thought to govern the target system. Often, such determinations can be made without recourse to elaborate derivations from theoretical first principles, but are enshrined in the notational conventions deployed by the formalism itself. (As a primitive analogy, consider how one might be able to spot a severe mistake in a mathematical derivation by checking that certain notational rules - e.g., regarding signs or the use of parentheses - were violated.) Importantly, the conventions of a particular mature mathematical formalism in a given scientific context, such as the creation/annihilation operator formalism in quantum many-body physics, add to, and are not merely the same as, the conventions of mathematics-at-large; for example, they might encompass locally applicable rules for the manipulation of the relevant mathematical symbols, where these rules are derived from, or otherwise systematically connected to, certain theoretical or methodological commitments. Examples of theoretical commitments that may be enshrined in a mature formalism would be certain fundamental laws of nature that are thought to govern the behaviour of the target system (and which one wants any model of that system to reflect), or an ontological commitment to the kinds of theoretical entities that a model may include as relevant. A mature mathematical formalism, one might say, embodies theoretical constraints and relationships in much the same way as, say, an orrery embodies the dynamics of the planetary system it represents (see the discussion at the end of Section 3.3.). ${ }^{17}$ The way in which certain constraints are incorporated into a formalism need not always be explicit; many mature formalisms involve an element of 'self-regulating conventionality', where such conventionality is not the result of any explicit attempt to conventionalise a practice', but arises from successive applications, for specific representational goals, in a way that is

role of mathematical formalism when he writes that 'a mathematical formalism is sought that is able to generate the relevant characteristics of the phenomena that should be explained or described' [Boumans 2005, p. 58, italics added]. However, mathematical formalism is still understood as 'one of the ingredients that should be integrated' (ibid., p. 17), rather than as an integrating force itself.

${ }^{17}$ While this is, of course, only an analogy, philosophers of mathematics, too, have recognised the parallels between mathematics and activities and phenomena in the material world. Thus, Imre Lakatos writes: "Mathematics, this product of human activity, "alienates itself" from the human activity which has been producing it. It becomes a living, growing organism, that acquires a certain autonomy from the activity which has produced it; it develops its own autonomous laws of growth, its own dialectic.' [Lakatos 1976, p. 146] 
'self-perpetuating and in need of no explicit, external introduction' [Netz 1999, p. $78 f.] .{ }^{18}$

Mature formalisms of the sort discussed here are not confined to, and indeed will usually go beyond, the notational devices standardly used in pure mathematics. In physics, especially, one finds many examples of formalisms that straddle the boundary between the mathematical and the merely diagrammatic. Some of these, such as Dirac's 'bra-ket' notation in quantum theory, which describes quantum states using angle brackets and vertical bars as notational devices (thus allowing for the quick evaluation of expectation values, or the spectral analysis of a Hamiltonian), are merely short-hand for more complex mathematical expressions. Others, such as the formalism of Feynman diagrams, have been developed with the goal of representing a potentially indefinite number of specific kinds of physical processes. Thus, a Feynman diagram in quantum electrodynamics consists of points ('vertices') and arrows (of different orientation) attached to the vertices, representing interacting electrons and positrons, as well as wavy lines signifying photons that may be emitted or absorbed. Enshrined in the formalism of Feynman diagrams are both rules for the construction of new diagrams (e.g., 'At every vertex, conservation of energy and momentum among the interacting particles is required'), as well as for the interpretation of the diagrams thus generated (e.g., 'Lines in intermediate stages in the diagram represent "virtual particles", which may "temporarily" violate the relativistic energy-momentum relation, but which are in-principle unobservable if they do not'). While the formalism of Feynman diagrams was developed on the basis of an overarching theoretical conception - according to which each diagram is to be interpreted as a contribution to the total amplitude for a (multiply realizable) quantum process - it has taken on 'a life of its own' in certain areas of high-energy physics, where it has developed from a mere shorthand to what one might call a notational 'lingua franca'. As physicists are keen to emphasise, 'Feynman diagrams provide deep physical insight into the nature of particle interactions', such as scattering processes, 'in addition to their value as a mathematical tool'. ${ }^{19}$

The gradual evolution from mere shorthands to powerful heuristic devices and theoretical tools, is characteristic of mature mathematical formalisms. As they undergo a process of elaboration, enrichment, and entrenchment, mature formalisms come to embody theoretical, ontological, and methodological commitments and assumptions; since these are enshrined in the formalism itself either in that the formalism allows only for some, but not all, possible processes and mechanisms to be represented, or because their theoretical character is cloaked behind purely notational rules - they are no longer readily obvious to either the novice or the proficient user. At the same time as formalisms

\footnotetext{
${ }^{18}$ This is not to say that formalisms do not include explicit rules and conventions, only that these do not exhaust the role of mature formalisms in scientific inquiry.

${ }^{19}$ See the entry 'Feynman diagram' on Wikipedia (7 March 2010 version, accessed 16 April 2010). It seems fair to say that, while not an authoritative source of knowledge, Wikipedia usually provides a good sense of what some of those who feel most strongly about a topic (in this case: theoretical physicists) tend to think.
} 
constrain what may be represented (and how), they also function as inferential and interpretative resources. ${ }^{20}$ While the rules governing, say, creation and annihilation operators shape how a many-body theorist will typically go about modelling complex phenomena, e.g. in the form of simple additive contributions to the overall Hamiltonian, they also often suggest 'concrete' interpretations of individual terms (as in the 'hopping' of electrons from one lattice site to another, discussed in Section 4.2.). It bears repeating that a mature mathematical formalism (in the sense discussed here) - even though it may afford its user considerable latitude in concrete applications - is infused with theoretical commitments, which determine, at least in part, which physically meaningful interpretations of a specific application are legitimate. This is precisely what makes mature formalisms so convenient to their users, who would otherwise have to settle on one amongst a plethora of prima facie permissible interpretations. Mature mathematical formalisms, thus, provide a crucial link between the realm of 'unconstrained' mathematics and the theoretical conception (or 'scientific image') of the physical domain of which the target system is a part. Michael Polanyi, in a different context, has commented on the dual character of mathematical formalism as both constraining and enabling, as well as on its role as a provider of access to inferential resources. On the one hand, Polanyi writes:

The process of reorganizing a conception for drawing new inferences from it can be formalized, by accepting as inferential operations certain rules for manipulating the symbols representing the states of affairs. [Polanyi 1962, p. 117]

On the other hand, routine formalization and acceptance of notational rules associated with formalisms, may well be a precondition for systematic inquiry into novel phenomena and explanations, since once it has been established, 'a mathematical formalism may be operated in ever new, uncovenanted ways, and force on our hesitant minds the expression of a novel conception'. [Polanyi 1962, p. 104] Mathematical formalisms, thus, not only occupy an interesting middle ground between 'global' ways of mathematically mapping the world-at-large and 'local' cases of mathematical moulding in concrete empirical contexts; they also play a vital role in allowing us to go beyond routine methods, by constructing new representational devices, thereby extending the reach of our representational practices to ever new domains.

\section{References}

[Baird 2004] Baird, Davis: Thing Knowledge: A Philosophy of Scientific Instruments, Berkeley: University of California Press 2004.

\footnotetext{
${ }^{20}$ As Marion Vorms argues in her contribution to this volume, the different formats and media in which models are presented, play a similar dual role in scientific representation.
} 
[Batterman 2002] Batterman, Robert W.: "Asymptotics and the Role of Minimal Models", British Journal for the Philosophy of Science 53 (2002) pp. 21-38.

[Boumans 1999] Boumans, Marcel: "Built-In Justification", in Mary S. Morgan and Margaret Morrison (eds.), Models as Mediators. Perspectives on Natural and Social Science, Cambridge: Cambridge University Press 1999, pp. 66-96.

[Boumans 2005] Boumans, Marcel, How Economicsts Model the World into Numbers, London: Routledge 2005.

[Cartwright 1983] Cartwright, Nancy, How the Laws of Physics Lie, Oxford: Oxford University Press 1983.

[Contessa 2007] Contessa, Gabriele: "Scientific Representation, Interpretation, and Surrogative Reasoning", Philosophy of Science 72 (2007) pp. 48-68.

[da Costa and French 2003] da Costa, Newton C.A., and Steven French, Science and Partial Truth: A Unitary Approach to Models and Scientific Reasoning, New York: Oxford University Press 2003.

[De Regt 2005] De Regt, Henk: "Scientific Realism in Action: Molecular Models and Boltzmanns's Bildtheorie", Erkenntnis 63 (2005) pp. 205230 .

[Ducheyne 2006] Ducheyne, Steffen: "Lessons from Galileo: The Pragmatic Model of Shared Characteristics of Scientific Representation", Philosophia Naturalis 43 (2006) pp. 214-234.

[Falkenburg 2007] Falkenburg, Brigitte: Particle Metaphysics: A Critical Account of Subatomic Reality, Berlin: Springer 2007.

[French 1999] French, Steven: "Models and Mathematics in Physics: The Role of Group Theory", in Jeremy Butterfield and Constantine Pagonis (eds.), From Physics to Philosophy, Cambridge: Cambridge University Press 1999, pp. 187-207.

[French 2003] French, Steven: "A Model-Theoretic Account of Representation (Or, I Don't Know Much about Art...but I Know It Involves Isomorphism", Philosophy of Science 79 (2003) pp. 1472-1483.

[Gelfert 2003] Gelfert, Axel: "Manipulative Success and the Unreal", International Studies in the Philosophy of Science 17 (2003) pp. 245263.

[Gelfert 2009] Gelfert, Axel: "Rigorous Results, Cross-Model Justification, and the Transfer of Empirical Warrant: The Case of Many-Body Models in Physics", Synthese 169 (2009) pp. 497-519.

[Goodman 1976] Goodman, Nelson: Languages of Art: An Approach to a Theory of Symbols, Indianapolis: Hackett 1976. 
[Hendry 1999] Hendry, Robin: "Theories and Models: the Interactive View", in Ray Paton and Irene Neilson (eds.), Visual Representations and Interpretations, Berlin: Springer 1999, pp. 121-130.

[Hendry and Psillos 2007] Robin F. Hendry and Stathis Psillos: "How to Do Things with Theories: An Interactive View of Language and Models in Science", in Jerzy Brzeziński, Andrzej Klawiter, Theo A.F. Kuipers, Krzysztof Łastowski, Katarzyna Paprzycka, \& Piotr Przybysz (eds.), The Courage of Doing Philosophy: Essays Dedicated to Leszek Nowak, Amsterdam: Rodopi 2007, pp. 123-157.

[Hesse 1953] Hesse, Mary: "Models in Physics", British Journal for the Philosophy of Science 4 (1953) pp. 198-214.

[Hesse 1963] Hesse, Mary: Models and Analogies in Science, London: Sheed and Ward 1963.

[Hughes 1996] Hughes, R.I.G.: "Models and Representation", Philosophy of Science 64 (Proceedings, PSA 1996) pp. S325-S336.

[Knuutttila 2010] Knuuttila, Tarja: "Modelling and Representing: An Artefactual Approach to Model-Based Representation", Studies in History and Philosophy of Science (this issue).

[Lakatos 1976] Lakatos, Imre: Proofs and Refutations: The Logic of Mathematical Discovery, Cambridge: Cambridge University Press 1976.

[Lewis 2004] Lewis, David: "Void and Object", in John Collins, Ned Hall, and L.A. Paul (eds.), Causation and Counterfactuals, Cambridge (Mass.): The MIT Press 2004.

[Morrison 1998] Morrison, Margaret: "Modelling Nature: Between Physics and the Physical World", Philosophia Naturalis 35 (1998) pp. 65-85.

[Morrison 1999] Morrison, Margaret: "Models as Autonomous Agents", in Mary S. Morgan and Margaret Morrison (eds.), Models as Mediators. Perspectives on Natural and Social Science, Cambridge: Cambridge University Press 1999, pp. 38-65.

[Morton 1993] Morton, Adam: "Mathematical Models: Questions of Trustworthiness", British Journal for the Philosophy of Science 44 (1993) pp. 659-674.

[Netz 1999] Netz, Raviel: The Shaping of Deduction in Greek Mathematics: A Study in Cognitive History, Cambridge: Cambridge University Press 1999.

[Pincock 2005] Pincock, Christopher: "Conditions on the Use of the Onedimensional Heat Equation", in G. Sica (ed.), Essays on the Foundations of Mathematics and Logic, Monza: Polimetrica 2005, pp. 67-79. 
[Pincock 2005b] Pincock, Christopher: "Overextending Partial Structures: Idealization and Abstraction", Philosophy of Science 72 (2005) pp. 1248-1259.

[Polanyi 1962] Polanyi, Michael: Personal Knowledge: Twoards a Post-Critical Philosophy, Chicago: The University of Chicago Press 1962.

[Redhead 1975] Redhead, Michael L.G.: "Symmetry in Intertheory Relations", Synthese 32 (1975) pp. 77-112.

[Rummel 1970] Rummel, Rudolph J., Applied Factor Analysis, Evanston: Northwestern University Press 1970.

[Suárez 1999] Suárez, Mauricio: "Theories, models, and representations", in Lorenzo Magnani, Nancy J. Nersessian, \& Paul Thagard (eds.), Model-based reasoning in scientific discovery, New York: Plenum Publishers 1999, pp. 75-83.

[Suárez 2003] Suárez, Mauricio: "Scientific Representation: Against Similarity and Isomorphism", International Studies in the Philosophy of Science 17 (2003) pp. 225-244.

[Suárez 2004] Suárez, Mauricio: "An Inferential Conception of Scientific Representation", Philosophy of Science 71 (2004) pp. 767-779.

[Suarez 2010] Suárez, Mauricio: "Scientific Representation", Philosophy Compass 5 (2010) pp. 91-101.

[Tarnow 1994] Tarnow, Eugen: "When Newton's cooling law doesn't hold", American Journal of Physics 62 (1994) pp. 89-90

[Teller 2001] Teller, Paul: "Twilight of the Perfect Model Model", Erkenntnis 55 (2001) pp. 393-415.

[Vorms 2010] Vorms, Marion: "Representing with Imaginary Models: Formats Matter", Studies in History and Philosophy of Science (this issue).

[Watson 1981] Watson, James: The Double Helix: A Person Account of the Discovery of the Structure of DNA, New York: Norton [1968] 1981.

[Weyl 1928] Weyl, Hermann: The Theory of Groups and Quantum Mechanics (transl. from the German 1928 edition), London: Methuen \& Co. 1931.

[Weyl 1968] Weyl, Hermann: Gesammelte Abhandlungen, Berlin: Springer 1968. 